\title{
Hall and ion slip effects on Unsteady MHD Convective Rotating flow of Nanofluids-Application in Biomedical Engineering
}

\author{
M. Veera Krishna ${ }^{1 *}$ (ID and Ali J. Chamkha ${ }^{2,3}$
}

* Correspondence:

veerakrishna_maths@yahoo.com

'Department of Mathematics, Rayalaseema University, Kurnool, Andhra Pradesh 518007, India Full list of author information is available at the end of the article

\begin{abstract}
There is an intense worldwide activity in the development of instrumentation for medical diagnosis and bioscreening based on biological labeling and detection of nanoparticles. Based on this profound observation, Hall and ion slip effects on magnetohydrodynamic (MHD) free convective rotating flow of nanofluids in a porous medium past a moving vertical semi-infinite flat plate are investigated. The equations for governing flow are solved analytically by perturbation approximation. The effects of various parameters on the flow are discussed through graphs and tables. The velocity increases with Hall and ion slip parameters. An increase in the convective parameter led to amplify the thermal boundary layer thickness, but when the heat generation parameter is taken into consideration, an opposite effect occurs. The skin friction coefficient increases with an increase in nanoparticle volume fraction and it reduces with increase in Hall and ion slip parameters. Outcomes disclose that the impact of thermal convection of nanoparticles has increased the temperature distribution, which helps in destroying the cancer cells during the drug delivery process.
\end{abstract}

Keywords: Hall and ion slip effects, Heat transfer, Porous medium, Nanofluids, Rotating frame

2010 Mathematics Subject Classification: 76E06, 76E07, 76S05, 81 V70, 82D80

\section{Introduction}

The homogeneous mixture of nanoparticles contains two liquids known as nanofluids. Nanoparticles operating in nanofluids are composed of carbon nanotubes, oxides, metals, and carbides. The base is made up of liquid oil, ethylene glycol, and water. The nanofluid was first proposed by Choi [1]. Nanofluids have applications in microelectronics, microfluidics, transportation, biomedical, X-rays, material processing, and scientific measurement. Buongiorno proposed an analytical model for convective transport in nanofluids, which incorporate the effects of Brownian diffusion and thermophoresis. These nanoparticles also hold a lot of significance in the areas of biological and medical applications. Some nanoparticles can bind many drugs, proteins, and target cancer cells. Since many nanoparticles have high atomic numbers that can

(c) The Author(s). 2020 Open Access This article is distributed under the terms of the Creative Commons Attribution 4.0 International License (http://creativecommons.org/licenses/by/4.0/), which permits unrestricted use, distribution, and reproduction in any medium, provided you give appropriate credit to the original author(s) and the source, provide a link to the Creative Commons license, and indicate if changes were made. 
produce heat, they lead to the treatment of tumor-selective photothermal therapy. Most of the nanoparticles can cure and help in targeting the deadly cancer cells. Flow through porous medium with nanoparticles has significant applications in biomedical science (such as drug delivery and cancer treatment to treat radiotherapy) and chemical engineering (transport of chemicals).

A major interest of convective heat transmission of nanofluids in sciences and engineering is incredibly significant. It is due to various varieties of cool devices like microelectronics and electronic gear and solar power technology. Water, ethylene glycol, and engine oil are heating or cooling agents and play a decisive job in thermal management of many industries with poor thermal conductivity. Recent investigations have given more attention towards thermal convection flows. The rate of thermal energy transmission from one point to another point enhances in several conductive and convection processes. The thermal energy vigorously depends on the variation of heat at the locomotion. However, in thermal radiation, energy transmission between two bodies depends upon absolute heat variation. Thermal radiation has so many applications in biomedical. Because of its application in biomedical and medical treatment, the outcome of thermal radiation with double diffusion has become an important research subject to many researchers. Infrared radiation is one of the most commonly used techniques for the treatment of heat in various parts of the body. Infrared radiation is made up of electromagnetic waves. It lies between the visible light and the microwave. It helps in correcting many skin-related problems. Any radiation entering the skin depends on the composition, vascularity, pigmentation, and wavelength of the radiation. Infrared radiation helps treat heat, directly heating blood vessels in the affected area of the body. It raises the blood supply in the body, which helps in adequate infection of the wound. It raises WBC and removes waste products. It is used in arthritic joints and other inflammatory conditions. Blood flows to different parts of the body. In skin friction, heat transmission occurs through radiation, evaporation, conduction, and circulation. It is known that infrared radiation is made up of electromagnetic waves, and so in the case of radiation heat transfer, the choice is transmitted by electromagnetic wave.

The porous medium is considered homogeneous and is present in local thermal equilibrium with isotropic nanoclides. Blood flow resistance for microbial biofilm and heat transmission around biological tissues, the concept of electronic preservation, hydrodynamic modeling of tissue-engineered materials, and general methods of antibiotic therapy. It describes how biofilm pores affect the hydrodynamics of the media. It describes a model of flow changes in brain aneurysms and recent advances in turbulence estimation methods, modeling passive mass transport processes, and also sheds light on cellular membranes. Further, it also has biochemical impacts and large-scale transport by the skin [2].

In many realistic applications that require a strong magnetic field, there is a need to think both the Hall and ion-slip currents because of the significant effect they have on the vector of the current density and transitively on the magnetic force idiom. The effects of Hall and ion-slip parameters on mixed convective electrically conducting nanofluid flow between two parallel concentric cylinders considering magnetic field has been discussed by Srinivasacharya and Shafeeurrahman [3]. Veera Krishna and Chamkha [4] investigated the diffusion-thermo, radiation-absorption and Hall and ion slip effects on MHD free convective rotating flow of nanofluids (Ag and TiO2) past a semi- 
infinite permeable moving plate with a constant heat source. Veera Krishna and Chamkha [5] discussed the MHD squeezing flow of a water-based nanofluid through a saturated porous medium between two parallel disks taking Hall current into account. Ram [6] investigated the effects of Hall and ion slip currents on MHD rotating free convective heat-generating flow. Seddeek [7] has discussed the effects of Hall and ion-slip currents and Heat transfer on magneto-micropolar fluid over a non-isothermal stretching sheet with suction and blowing. Seddeek and Abdelmeguid [8] discussed the boundary layer analysis which is used to the effects of Hall and ion-slip currents on steady magnetomicropolar fluid over a horizontal plate. Jha and Apere [9] investigated unsteady MHD Couette flow of a Newtonian fluid between two rotating parallel plates taking hall and ion-slip currents. Uddin and Kumar [10] discussed Hall and ion-slip effect on the thickness of the boundary layer flow of a micropolar fluid over a wedge. Ellahi et al. [11] conducted a theoretical study of the problem of the peristaltic flow of Jeffrey fluid in a nonuniform rectangular duct under the effects of Hall and ion slip. Bhatti et al. [12] discussed the effect of Hall and ion slip on peristaltic blood flow of Eyring Powell in a non-Uniform Porous two-dimensional channel under long wavelength approximation of zero Reynolds number. Veera Krishna and Subba Reddy [13] investigated the transient MHD flow of a reactive second-grade fluid through a porous medium between two infinitely long horizontal parallel plates. Jitendra and Srinivasa [14] investigated Hall and ion slip effects on the convective flow of a rotating fluid. Dileep and Priyanka [15] discussed the Hall effects on MHD viscous electrically conducting fluid flow and heat transfer in a parallel plate channel partially filled with a porous medium with an inclined magnetic field in a rotating system. Veera Krishna et al. [16] discussed the Hall effects on unsteady MHD oscillatory free convective flow of second-grade fluid through porous medium between two vertical plates. Veera Krishna and Chamkha [17] have delivered the unsteady MHD flow of second-grade fluid through porous medium with ramped wall temperature and ramped surface concentration with Hall effects. Veera Krishna et al. [18] have investigated heat and mass transfer on MHD chemically reacting flow of micropolar fluid through a porous medium with Hall effects. Veera Krishna et al. [19] discussed the Soret and Joule effects of MHD mixed convective flow of an incompressible and electrically conducting viscous fluid past an infinite vertical porous plate considering Hall effects. Veera Krishna et al. [20] have discussed Hall effects on MHD peristaltic flow of Jeffrey fluid through porous medium in a vertical stratum. Sara and Bhatti [21] investigated MHD peristaltic flow of a non-Newtonian nanofluid with chemical reaction, Hall and ion-slip currents. Bhatti et al. [22] discussed the effects of heat transfer and Hall current on the sinusoidal motion of solid particles through a planar channel. Waqas et al. [23] investigated to visualize the flow of modified second grade nanofluid with heat, motile microorganisms, and mass transfer rates over stretching surface. Sheikholeslami and Bhatti [24] analyzed magnetic nanofluid transportation under the effect of a non-uniform magnetic field in a porous cavity. The effects of heat and mass transfer on free convective flow of micropolar fluid were studied over an infinite vertical porous plate in the presence of an inclined magnetic field with a constant suction velocity and taking Hall current into account have been discussed by Veera Krishna et al. [25]. Veera Krishna and Chamkha [26] have discussed the systematic solution of time-dependent mean velocity on MHD peristaltic rotating flow of an electrically conducting couple stress fluid in a uniform elastic porous channel. 
The aforementioned studies and literature survey bear witness that the analysis of Hall and ion effects of a viscous incompressible electrically conducting nanofluid in an infinite vertical plate with rotation effect has not been presented yet. In order to fill the gap of the existing literature, the Hall and ion slip effects on the unsteady MHD free convective rotating flow of nanofluids in a porous medium past infinite vertical flat plate have been undertaken and discussed.

\section{Formulation and solution of the problem}

We have considered Hall and ion slip effects on the unsteady free convective flow of nanofluids $\left(\mathrm{Cu}\right.$ and $\left.\mathrm{TiO}_{2}\right)$ of ambient temperature $T_{\infty}$ over a vertical semi-infinite moving plate entrenched in a homogeneous porous medium under thermal buoyancy effect with a constant heat source and convective boundary conditions. We assumed both the fluid phase and nanoparticles are in a thermal equilibrium state. Also, we assume a uniform shape and sized nanoparticles. The nanoparticles of a smaller size than that of the matrix pores are suspended in nanofluid using either surfactant or surface change technology, preventing the agglomeration and deposition of these on the porous matrix. The porous medium is considered as homogeneous as well as isotropic and is in local thermal equilibrium with the nanofluid. The thermophysical properties of pure water, $\mathrm{Cu}$, and titanium oxide nanoparticles are taken to be constant at a reference temperature $\left(25^{\circ} \mathrm{C}\right)$ and are given in Table 1 [27].

Figure 1 portrays the physical model of the problem. The flow is assumed to be in the $x$-direction which is obtained along the plate in the ascendant direction and $z$-axis is normal to it. The entire system rotates with an angular velocity $\Omega$ about $z$-axis. An unvarying peripheral magnetic field $B_{0}$ is taken to be acting along the $z$-axis. There is no applied voltage $(E=0)$. The induced magnetic field is tiny compared to the external magnetic field.

Due to semi-infinite plate surface assumption, all the variables are functions of $z$ and time $t$ only. Under the boundary layer approximations, the basic equations that describe the physical situation are given by

$$
\begin{aligned}
& \frac{\partial u}{\partial x}+\frac{\partial v}{\partial y}=0 \\
& \frac{\partial u}{\partial t}+w \frac{\partial u}{\partial z}-2 \Omega v=\frac{\mu_{n f}}{\rho_{n f}} \frac{\partial^{2} u}{\partial z^{2}}+\frac{B_{0} J_{y}}{\rho_{n f}}-\frac{\mu_{n f} u}{\rho_{n f} k}+g \beta_{n f}\left(T-T_{\infty}\right) \\
& \frac{\partial v}{\partial t}+w \frac{\partial v}{\partial z}+2 \Omega u=\frac{\mu_{n f}}{\rho_{n f}} \frac{\partial^{2} v}{\partial z^{2}}-\frac{B_{0} J_{x}}{\rho_{n f}}-\frac{\mu_{n f} v}{\rho_{n f} k}
\end{aligned}
$$

Table 1 Thermo-physical properties of regular fluid and nanoparticles

\begin{tabular}{llll}
\hline Thermo-physical properties & Regular fluid (water) & $\mathrm{Cu}$ & $\mathrm{TiO}_{2}$ \\
\hline$C_{p}(\mathrm{~J} / \mathrm{kg} \mathrm{K})$ & 4179 & 385 & 686.2 \\
$\rho\left(\mathrm{kg} / \mathrm{m}^{3}\right)$ & 997.1 & 8933 & 4250 \\
$k(\mathrm{~W} / \mathrm{m} \mathrm{K})$ & 0.613 & 400 & 8.9538 \\
$\beta \times 10^{-5}(1 / \mathrm{K})$ & 21 & 1.67 & 0.9 \\
\hline
\end{tabular}




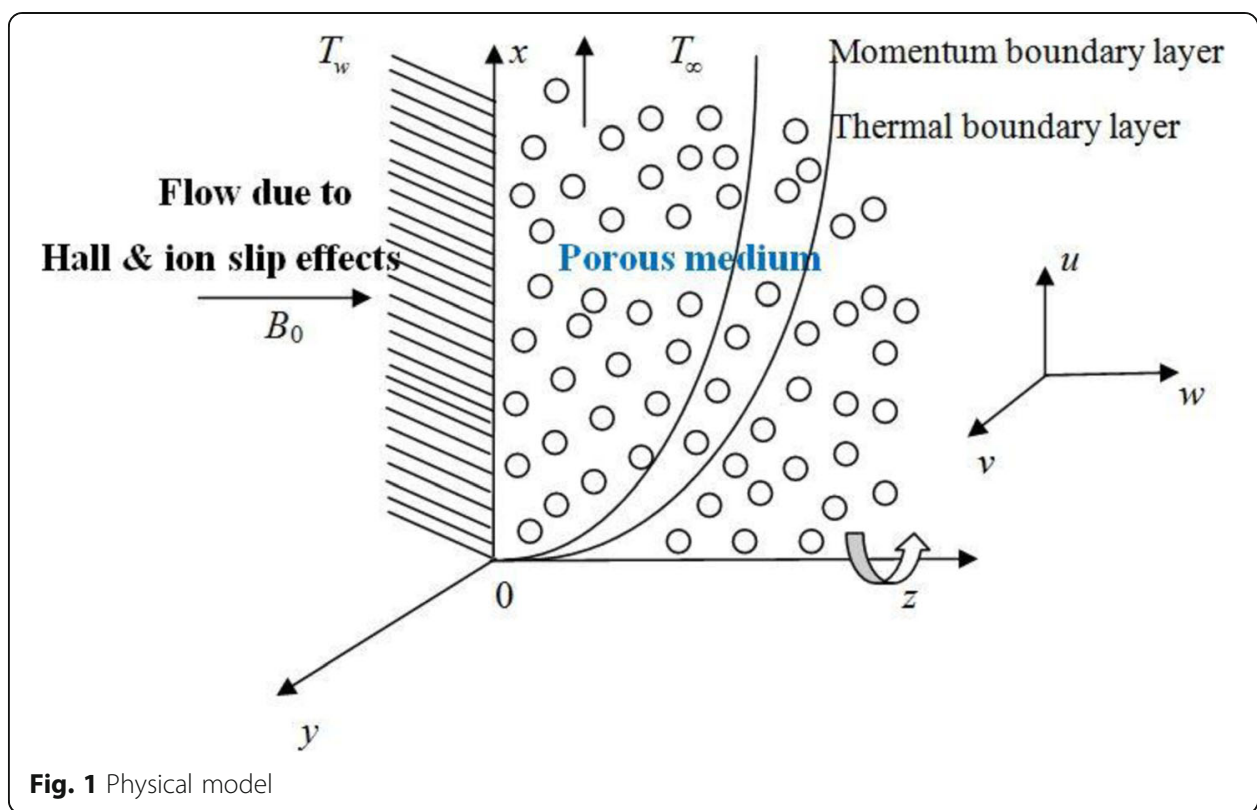

$$
\frac{\partial T}{\partial t}+w \frac{\partial T}{\partial z}=\alpha_{n f} \frac{\partial^{2} T}{\partial z^{2}}-\frac{Q}{\left(\rho C_{p}\right)_{n f}}\left(T-T_{\infty}\right)
$$

The boundary conditions are

$$
\begin{aligned}
& u=v=0, T=T_{\infty} \text { for } t \leq 0 \\
& \left.u=U_{r}\left(1+\frac{\varepsilon}{2}\left(e^{i n t}+e^{-i n t}\right)\right), v=0,-K_{n f} \frac{\partial T}{\partial z}=h_{f}\left(T_{w}-T_{\infty}\right) \text { at } z=0\right\} \text { for } t>0
\end{aligned}
$$

The electron-atom collision frequency is assumed very high, so that Hall and ion-slip currents cannot be neglected. Hence, the Hall and ion-slip currents give rise to the velocity in $y$-direction. When the strength of the magnetic field is very large, the generalized Ohm's law is [28]

$$
\left.J=\sigma(E+V \times B)-\frac{\omega_{e} \tau_{e}}{B_{0}}(J \times B)+\frac{\omega_{e} \tau_{e} \beta_{i}}{B_{0}^{2}}((J \times B) \times B)\right)
$$

The component equations of (7) are

$$
\begin{aligned}
& \left(1+\beta_{i} \beta_{e}\right) J_{x}+\beta_{e} J_{y}=\sigma B_{0} v \\
& \left(1+\beta_{i} \beta_{e}\right) J_{y}-\beta_{e} J_{x}=-\sigma B_{0} u
\end{aligned}
$$

On solving Eqs. (8) and (9), we get

$$
\begin{aligned}
& J_{x}=\sigma B_{0}\left(\alpha_{2} u+\alpha_{1} v\right) \\
& J_{y}=-\sigma B_{0}\left(\alpha_{2} v-\alpha_{1} u\right)
\end{aligned}
$$

Substituting the Eqs. (10) and (11) in (3) and (2), respectively, we obtain 


$$
\begin{aligned}
& \frac{\partial u}{\partial t}+w \frac{\partial u}{\partial z}-2 \Omega v=\frac{\mu_{n f}}{\rho_{n f}} \frac{\partial^{2} u}{\partial z^{2}}+\frac{\sigma B_{0}^{2}\left(\alpha_{2} v-\alpha_{1} u\right)}{\rho_{n f}}-\frac{\mu_{n f} u}{\rho_{n f} k}+g \beta_{n f}\left(T-T_{\infty}\right) \\
& \frac{\partial v}{\partial t}+w \frac{\partial v}{\partial z}+2 \Omega u=\frac{\mu_{n f}}{\rho_{n f}} \frac{\partial^{2} v}{\partial z^{2}}-\frac{\sigma B_{0}^{2}\left(\alpha_{2} u+\alpha_{1} v\right)}{\rho_{n f}}-\frac{\mu_{n f} v}{\rho_{n f} k}
\end{aligned}
$$

The effective density, thermal diffusivity, heat capacitance, thermal conductivity [29], thermal expansion coefficient, and effective dynamic viscosity [30] of the nanofluid are

$$
\begin{aligned}
& \rho_{n f}=(1-\phi) \rho_{f}+\phi \rho_{s} \\
& \alpha_{n f}=\frac{K_{n f}}{\left(\rho C_{p}\right)_{n f}} \\
& \left(\rho C_{p}\right)_{n f}=(1-\phi)\left(\rho C_{p}\right)_{f}+\phi\left(\rho C_{p}\right)_{s} \\
& \frac{k_{n f}}{k_{f}}=\frac{\left(k_{s}+2 k_{f}\right)-2 \phi\left(k_{f}-k_{s}\right)}{\left(k_{s}+2 k_{f}\right)+2 \phi\left(k_{f}-k_{s}\right)} \\
& (\rho \beta)_{n f}=(1-\phi)(\rho \beta)_{f}+\phi(\rho \beta)_{s} \\
& \mu_{n f}=\frac{\mu_{f}}{(1-\phi)^{2.5}} \\
& w=-w_{0}
\end{aligned}
$$

where $w_{0}$ represents the normal velocity at the plate, which is positive for suction and negative for injection. The following dimensionless variables

$$
\begin{aligned}
& u *=\frac{u}{U_{r}}, \nu *=\frac{v}{U_{r}}, z *=\frac{z U_{r}}{v_{f}}, t *=\frac{t U_{r}^{2}}{v_{f}}, n *=\frac{n v_{f}}{U_{r}^{2}}, \theta=\frac{\left(T-T_{\infty}\right)}{\left(T_{w}-T_{\infty}\right)}, \gamma=\frac{h_{f} v_{f}}{K_{f} U_{r}} \\
& R=\frac{\Omega v_{f}}{U_{r}^{2}}, M=\frac{B_{0}}{U_{r}} \sqrt{\frac{\sigma v_{f}}{\rho_{f}}}, \operatorname{Pr}=\frac{v_{f}}{\alpha_{f}}, S=\frac{w_{0}}{U_{r}}, K=\frac{k U_{r}^{2}}{v_{f}^{2}}, Q_{H}=\frac{Q v_{r}^{2}}{U_{r}^{2} k_{f}}
\end{aligned}
$$

Using non-dimensional variables, Eqs. (2)-(4) yields (dropping asterisks),

$$
\begin{aligned}
& a_{1}\left(\frac{\partial u}{\partial t}-S \frac{\partial u}{\partial z}-2 R v\right)=a_{3} \frac{\partial^{2} u}{\partial z^{2}}+M^{2}\left(\alpha_{2} v-\alpha_{1} u\right)-\frac{u}{K}+a_{2} \theta \\
& a_{1}\left(\frac{\partial v}{\partial t}-S \frac{\partial v}{\partial z}+2 R u\right)=a_{3} \frac{\partial^{2} v}{\partial z^{2}}-M^{2}\left(\alpha_{2} u+\alpha_{1} v\right)-\frac{v}{K} \\
& a_{4}\left(\frac{\partial \theta}{\partial t}-S \frac{\partial \theta}{\partial z}\right)=\frac{1}{\operatorname{Pr}}\left(\frac{k_{n f}}{k_{f}} \frac{\partial^{2} \theta}{\partial z^{2}}-Q_{H} \theta\right)
\end{aligned}
$$

The velocity characteristic $U_{r}$ is as [31]

$$
U_{r}=\left(g \beta_{f}\left(T_{w}-T_{\infty}\right) v_{f}\right)^{1 / 3}
$$

The non-dimensional boundary conditions are

$$
u=v=0, \theta=0 \text { for } t \leq 0
$$




$$
\begin{aligned}
& u=1+\frac{\varepsilon}{2}\left(e^{i n t}+e^{-i n t}\right), v=0, \frac{d \theta}{d z}=-\gamma(1-\theta(z)) \text { at } z=0 \\
& u \rightarrow 0, v \rightarrow 0, \theta \rightarrow 0 \text { for } t>0
\end{aligned}
$$

We now combining the Eqs. (21) and (21) $(q=u+i v)$, we get

$$
a_{1}\left(\frac{\partial q}{\partial t}-S \frac{\partial q}{\partial z}+2 i R q\right)=a_{3} \frac{\partial^{2} q}{\partial z^{2}}-\left(M^{2}\left(\alpha_{1}+i \alpha_{2}\right)+\frac{1}{K}\right) q+a_{2} \theta
$$

The boundary conditions (24) and (25) are as follows:

$$
\begin{aligned}
& q=0, \theta=0, \text { for } t \leq 0 \\
& \left.\begin{array}{l}
q=1+\frac{\varepsilon}{2}\left(e^{i n t}+e^{-i n t}\right), \frac{d \theta}{d z}=-\gamma[(1-\theta(z))] \text { at } z=0 \\
q \rightarrow 0, \theta \rightarrow 0 \text { as } z \rightarrow \infty
\end{array}\right\} \text { for } t>0
\end{aligned}
$$

To find the analytical solutions of the PDE (23), (26) with the boundary conditions (27), (28), then, $q$ and $\theta$ as [32],

$$
\begin{aligned}
& q(z, t)=q_{0}+\frac{\varepsilon}{2}\left(e^{i n t} q_{1}(z)+e^{-i n t} q_{2}(z)\right) \\
& \theta(z, t)=\theta_{0}+\frac{\varepsilon}{2}\left(e^{i n t} \theta_{1}(z)+e^{-i n t} \theta_{2}(z)\right)
\end{aligned}
$$

for $\varepsilon(<<1)$.

Replacing Eqs. (29) and (30) into (26) and (23), respectively, and equating harmonic and non-harmonic terms (neglect the higher poers of $\varepsilon$ ), we obtain

$$
\begin{aligned}
& a_{3} \frac{d^{2} q_{0}}{d z^{2}}+S_{3} a_{1} \frac{d q_{0}}{d z}-\left(M^{2}\left(\alpha_{1}+i \alpha_{2}\right)+\frac{1}{K}+2 i R a_{1}\right) q_{0}+a_{2} \theta_{0}=0 \\
& a_{3} \frac{d^{2} q_{1}}{d z^{2}}+S a_{1} \frac{d q_{1}}{d z}-\left(M^{2}\left(\alpha_{1}+i \alpha_{2}\right)+\frac{1}{K}+i(2 R+n) a_{1}\right) q_{1}+a_{2} \theta_{1}=0 \\
& a_{3} \frac{d^{2} q_{2}}{d z^{2}}+S a_{1} \frac{d q_{2}}{d z}-\left(M^{2}\left(\alpha_{1}+i \alpha_{2}\right)+\frac{1}{K}+i(2 R-n) a_{1}\right) q_{2}+a_{2} \theta_{2}=0 \\
& \frac{k_{n f}}{k_{f}} \frac{d^{2} \theta_{0}}{d z^{2}}+\operatorname{Pr} S a_{4} \frac{d \theta_{0}}{d z}-Q_{H} \theta_{0}=0 \\
& \frac{k_{n f}}{k_{f}} \frac{d^{2} \theta_{1}}{d z^{2}}+\operatorname{Pr} S a_{4} \frac{d \theta_{1}}{d z}-\left(\text { in } \operatorname{Pr} a_{4}+Q_{H}\right) \theta_{1}=0 \\
& \frac{k_{n f}}{k_{f}} \frac{d^{2} \theta_{2}}{d z^{2}}+\operatorname{Pr} S a_{4} \frac{d \theta_{2}}{d z}-\left(i n \operatorname{Pr} a_{4}-Q_{H}\right) \theta_{2}=0
\end{aligned}
$$

The corresponding boundary conditions are as follows:

$$
\begin{aligned}
& q_{0}=q_{1}=q_{2}=1, \frac{d \theta_{0}}{d z}=-\gamma\left(1-\theta_{0}(z)\right), \frac{d \theta_{1}}{d z}=\gamma \theta_{1}(z), \frac{d \theta_{2}}{d z}=\gamma \theta_{2}(z) \text { at } z=0 \\
& q_{0} \rightarrow 0, q_{1} \rightarrow 0, q_{2} \rightarrow 0, \theta_{0} \rightarrow 0, \theta_{1} \rightarrow 0, \theta_{2} \rightarrow 0 \text { as } z \rightarrow \infty
\end{aligned}
$$

Solving Eqs. (31)-(36) under the boundary conditions (37)-(38), we obtain velocity and temperature as 


$$
\begin{aligned}
& q=A_{1} e^{-m_{1} z}+\left(1-A_{1}\right) e^{-m_{2} z}+\frac{\varepsilon}{2}\left(e^{-m_{3} z+i n t}+e^{-m_{4} z-i n t}\right) \\
& \theta=\frac{\gamma}{m_{1}+\gamma} e^{-m_{1} z}
\end{aligned}
$$

The skin-friction coefficient $C_{f}$ and the local Nusselt number $N u$ are

$$
\begin{aligned}
C_{f} & =\frac{\left(T_{w}\right)_{z=0}}{\rho_{f} U_{t}^{2}}=a_{3}\left(\frac{d q}{d z}\right)_{z=0} \\
& =-a_{3}\left(A_{1} m_{1}+\left(1-A_{1}\right) m_{2}+\frac{\varepsilon}{2}\left(m_{3} e^{i n t}+m_{4} e^{-i n t}\right)\right) \\
N u & =\frac{x\left(\frac{\partial T}{\partial z}\right)_{z=0}}{T_{w}-T_{\infty}}=-\frac{k_{n f}}{k_{f}} \operatorname{Re}_{x}\left(\frac{d \theta}{d z}\right)_{z=0}
\end{aligned}
$$

Where $\operatorname{Re}_{x}=\frac{U_{r} x}{v_{f}}$ is the local Reynolds number. Thus

$$
\frac{N u}{\operatorname{Re}_{x}}=-\frac{k_{n f}}{k_{f}}\left(\frac{d \theta}{d z}\right)_{z=0}
$$

Where, $\quad \alpha_{1}=\frac{1+\beta_{e} \beta_{i}}{\left(1+\beta_{e} \beta_{i}\right)^{2}+\beta_{e}^{2}}, \alpha_{2}=\frac{\beta_{e}}{\left(1+\beta_{e} \beta_{i}\right)^{2}+\beta_{e}^{2}}, A_{1}=\frac{\gamma(1-\phi)^{2.5} a_{2}}{\left(m_{1}+\gamma\right)\left(m_{1}^{2}-S_{1} m_{1}-B_{1}\right)}, \quad a_{1}=1-\phi+\phi\left(\frac{\rho_{s}}{\rho_{f}}\right)$, $a_{2}=1-\phi+\phi\left(\frac{(\rho \beta)_{s}}{(\rho \beta)_{f}}\right), a_{3}=\frac{1}{(1-\phi)^{2.5}}, a_{4}=1-\phi+\phi\left(\frac{\left(\rho C_{p}\right)_{s}}{\left(\rho C_{p}\right)_{f}}\right), \quad a_{5}=\frac{\operatorname{Pr} k_{f} a_{4}}{k_{n f}(1-\phi)^{2.5} a_{1}}, B_{1}=M_{1}+i R_{1}$, $B_{2}=M_{1}+i\left(R_{1}+n_{1}\right), B_{3}=M_{1}+i\left(R_{1}-n_{1}\right), M_{1}=\left(M^{2}\left(\alpha_{1}+i \alpha_{2}\right)+\frac{1}{K}\right)(1-\phi)^{2.5}, m_{1}=\frac{1}{2}\left[S_{1}\right.$ $\left.a_{5}+\sqrt{\left(S_{1} a_{5}\right)^{2}+4 Q_{H} \frac{k_{f}}{k_{n f}}}\right], m_{2}=\frac{1}{2}\left[S_{1}+\sqrt{\left(S_{1}\right)^{2}+4 B_{1}}\right], m_{3}=\frac{1}{2}\left[S_{1}+\sqrt{\left(S_{1}\right)^{2}+4 B_{2}}\right], m_{4}$ $=\frac{1}{2}\left[S_{1}+\sqrt{\left(S_{1}\right)^{2}+4 B_{3}}\right], n_{1}=n(1-\phi)^{2.5} a_{1}, R_{1}=R(1-\phi)^{2.5} a_{1}, S_{1}=S(1-\phi)^{2.5} a_{1}$,

\section{Results and discussion}

We become aware that solutions (39) and (40) move towards the solutions for the constant surface temperature as $\gamma \rightarrow \infty$ from the boundary condition (28), which gives $\theta$ (0) $=1$ as $\gamma \rightarrow \infty$. Further, it is worth mentioning that Eqs. (39) and (40) are reduced to those of Hamad and Pop [31] when $\beta_{e}=\beta_{i}=0, K \rightarrow \infty$ (absence of Hall and ion slip effects and non-porous medium) and $\gamma \rightarrow \infty$ (constant surface temperature). We have to discuss the heat transfer characteristics of the flow with nanoparticles that are presented in Figs. 2, 3, and 4 and in Tables 2 and 3. According to Oztop and Abu-Nada [27], we have taken the range of nanoparticle volume fraction $0 \leq \phi \leq 0.2$ and Prandtl number $\operatorname{Pr}=6.785$. We fixed $n=10, n t=\pi / 2$ and $\varepsilon=0.001$, while $\phi=0.05, \beta_{e}=0.3, \beta_{i}=$ $1, R=0.5, K=0.5, M=0.5, S=1, Q_{H}=1$, and $\gamma=2$ are varied over a range.

Figure 2a illustrates the influence of the magnetic field parameter $M$ on the velocity distribution for $\mathrm{Cu}$-water and $\mathrm{TiO}_{2}$-water nanofluids. The velocity across the boundary layer reduces with an increase in the magnetic field parameter $M$ and decreases asymptotically to zero at the boundary, which leads to a reduction in the layer thickness due to Lorentz force.

For different values of the permeability parameter $K$, the velocity distribution on the porous wall is plotted in Fig. $2 \mathrm{~b}$ for $\mathrm{Cu}$-water and $\mathrm{TiO}_{2}$-water. It is obvious that the increased values of $K$ tend to increase the velocity on the porous wall and so enhance the momentum boundary layer thickness. Figure $2 \mathrm{c}$ depicts the velocity with the different values of Hall parameter $\beta_{e}$ for $\mathrm{Cu}$-water and $\mathrm{TiO}_{2}$-water. Increased values of $\beta_{e}$ 

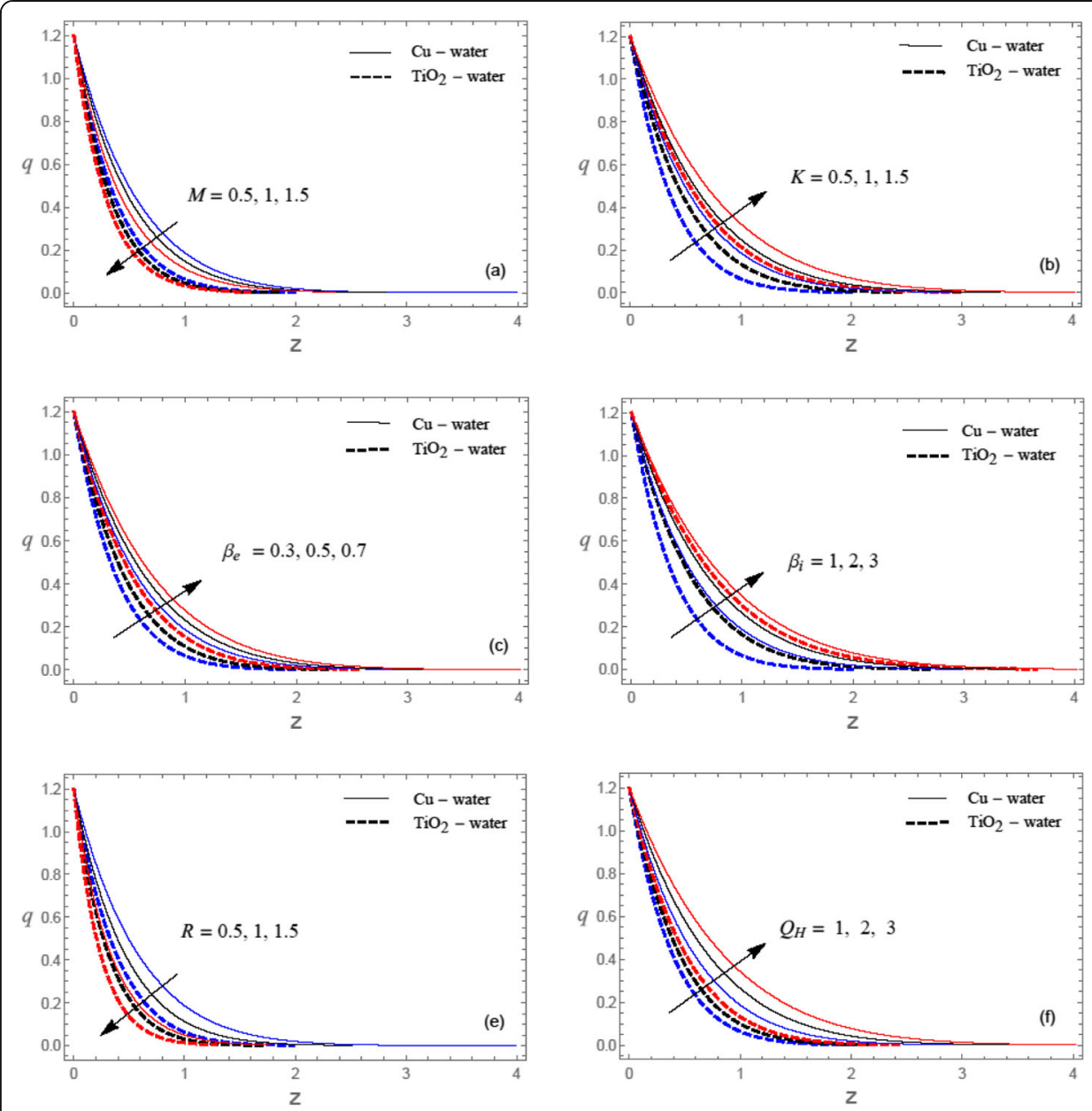

Fig. 2 a-f The velocity profiles with $M, K, \beta_{e}, \beta_{i l} R$, and $Q_{H}$

tend to increase the velocity and so enhance the momentum boundary layer thickness. The effect of ion slip parameter $\beta_{i}$ on velocity as observed in Fig. $2 \mathrm{~d}$ is similar to the effect of Hall current. The opposite effect is noticed on increasing rotation parameter $R$, i.e., increasing the rotation reduces the momentum boundary layer thickness (Fig. 2e). Figure $2 \mathrm{f}$ denotes the velocity profile with effect from the heat source parameter $Q_{H}$. The magnitude of the velocity increases with increasing $Q_{H}$ throughout the fluid region.

Figure 3a and $\mathrm{b}$ demonstrates the effect of the suction/injection parameter $S$ on the fluid velocity. The velocity across the boundary layer decreases for $S(>0)$ whereas increases for $S(<0)$ for both nanofluids with nanoparticles $\mathrm{Cu}$ and $\mathrm{TiO}_{2}$. So also as $S$ increases, the velocity still approaches the same asymptotic value for large values of $z$. Thus, the thickness of the boundary layer decreases with an increase in the suction parameter $S(>0)$. A reversal of this trend is noticed with injection parameter for $S(<0)$. Figure $3 \mathrm{c}$ illustrates the velocity for an assortment of the nanoparticle volume fraction parameter $\phi$. Hence, the velocity of the fluid across the boundary layer decreases with the increase of $\phi$. Figure $3 \mathrm{~d}$ represents the velocity distribution with the different values of convection parameter $\gamma$ for $\mathrm{Cu}$-water and $\mathrm{TiO}_{2}$-water. Increased values of $\gamma$ tend to increase the velocity and so accelerate the momentum boundary layer thickness. 

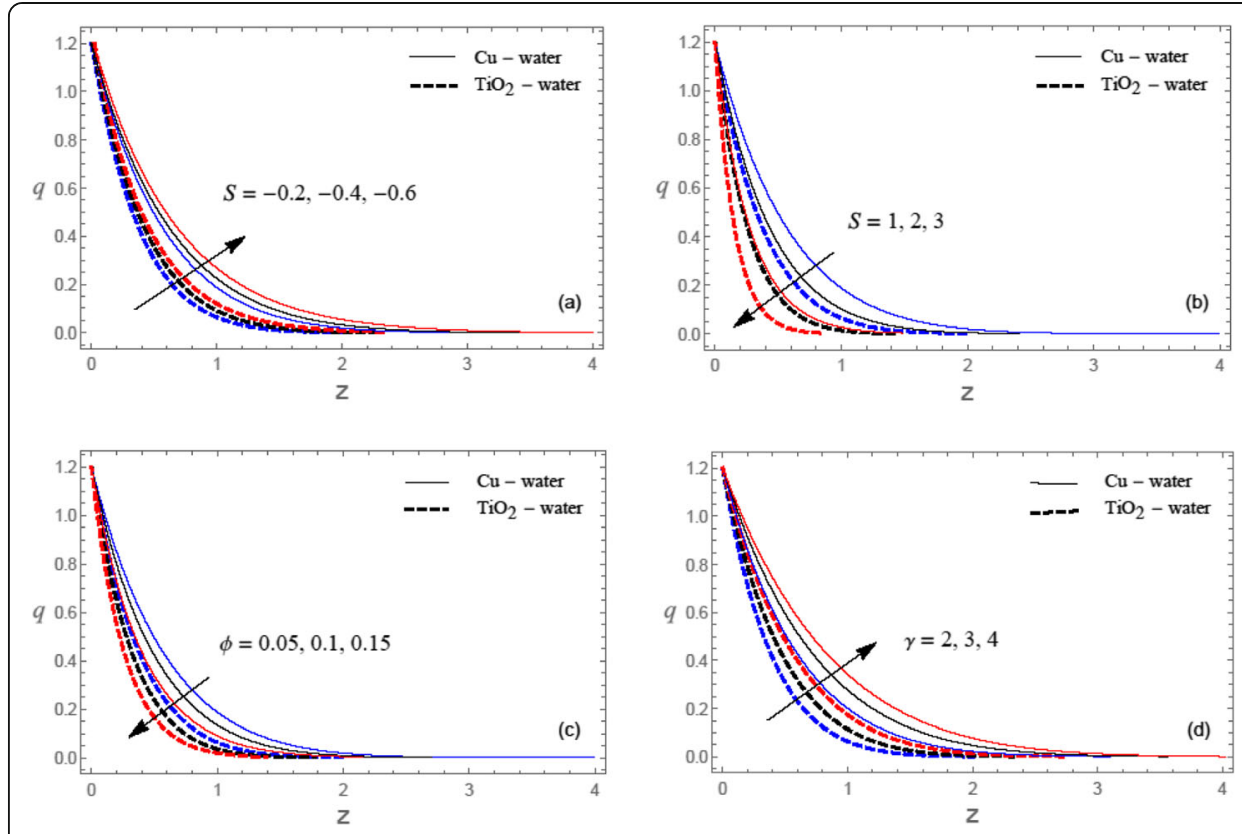

Fig. 3 a-d The velocity profiles against $S(<0), S(>0), \varphi$, and $\gamma$

Figure 4a displays the temperature profiles for various values of the heat generation parameter $Q_{\mathrm{H}}$ for both nanofluids with nanoparticles $\mathrm{Cu}$ and $\mathrm{TiO}_{2}$. The temperature in the boundary layer region decreases with the increase in the heat generation parameter $Q_{\mathrm{H}}$, and as a consequence, the thermal boundary layer thickness decreases. These side views satisfy the far field boundary conditions asymptotically, which bear the mathematical results obtained.

Figure $4 \mathrm{~b}$ presents a typical profile for temperature with the convective parameter $\gamma$ for both nanofluids. The temperature increases on increasing $\gamma$ in the boundary layer region and is maximum at the surface of the plate for both nanoparticles. Thus, by escalating $\gamma$, thermal boundary layer thickness enhances. So, we can interpret that the rate of heat transfer increases with increase in convective parameter $\gamma$. Figure $4 \mathrm{c} \mathrm{dem-}$ onstrates the variation of suction parameter $S$ on temperature for both nanofluids. The magnitude of the temperature reduces with increasing suction parameter $S$ and then thermal boundary layer thickness retarded throughout the fluid region. The influence of nanoparticle volume fraction parameter $\phi$ on the temperature is shown in Fig. $4 \mathrm{~d}$ for $\mathrm{Cu}$-water and $\mathrm{TiO}_{2}$-water. The temperature profile increases with the increase in nanoparticle volume fraction parameter $\phi$. Hence, the thermal boundary layer thickness improves and tends asymptotically to zero as the distance from the boundary is enhanced.

The variation of the skin friction coefficient $C_{f}$ and the Nusselt number $N u / \mathrm{Re}_{x}$ with $M, K, \beta_{e}, \beta_{i}, R, \gamma, Q_{H}, S$, and $\phi$ are shown in Tables 2 and 3, respectively. Table 2 shows that the skin friction coefficient $C_{f}$ decreases with increasing parameters $K, \beta_{e}, \beta_{i}$, and $Q_{H}$ whereas the skin friction coefficient increases with increasing $M, R, S, \gamma$, and $\phi$ for both nanofluids with nanoparticles $\mathrm{Cu}$ and $\mathrm{TiO}_{2}$. Also, from Table 3, the Nusselt number increases with the increase in all parameters $\gamma, Q_{H}, S$, and $\phi$ for both nanofluids with nanoparticles $\mathrm{Cu}$ and $\mathrm{TiO}_{2}$. The variation of Nusselt number is much more 

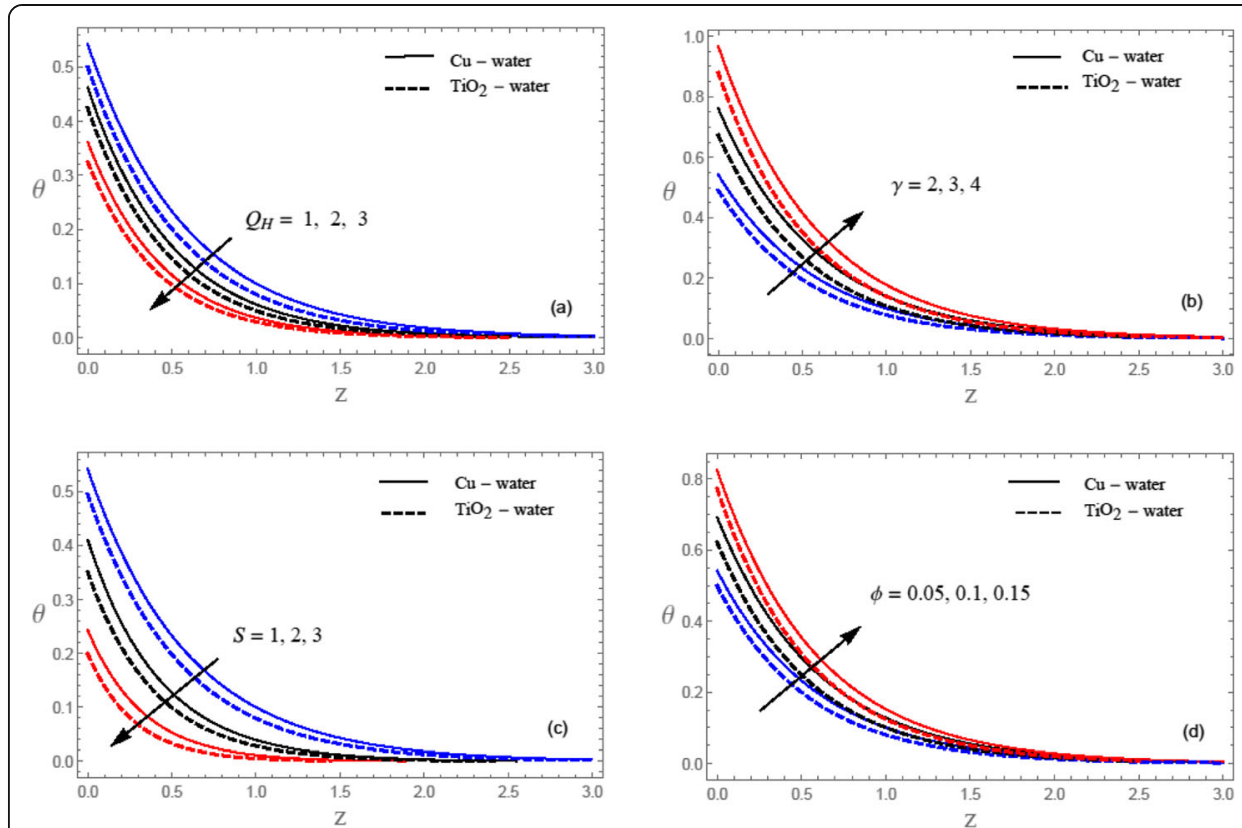

Fig. 4 a-d The temperature profile against $Q_{H} \gamma, S$, and $\varphi$

Table 2 Skin friction coefficient $\left(C_{f}\right)$

\begin{tabular}{|c|c|c|c|c|c|c|c|c|c|c|}
\hline$M$ & $K$ & $\beta_{e}$ & $\beta_{i}$ & $R$ & $\gamma$ & $Q_{H}$ & 5 & $\varphi$ & $\mathrm{Cu}$-water & $\mathrm{TiO}_{2}$-water \\
\hline 0.5 & \multirow[t]{3}{*}{0.5} & 1 & 0.3 & 0.5 & 2 & 1 & 1 & 0.05 & 2.61072 & 2.46031 \\
\hline 1.0 & & & & & & & & & 2.72080 & 2.57305 \\
\hline 1.5 & & & & & & & & & 2.89204 & 2.74765 \\
\hline & 1.0 & & & & & & & & 2.28173 & 2.11971 \\
\hline & \multirow[t]{15}{*}{1.5} & & & & & & & & 2.15766 & 1.98908 \\
\hline & & 2 & & & & & & & 2.58795 & 2.43693 \\
\hline & & 3 & & & & & & & 2.55557 & 2.41907 \\
\hline & & & 0.5 & & & & & & 2.55254 & 2.40252 \\
\hline & & & 0.7 & & & & & & 2.49885 & 2.38479 \\
\hline & & & & 1.0 & & & & & 2.67358 & 2.50811 \\
\hline & & & & 1.5 & & & & & 2.76488 & 2.57943 \\
\hline & & & & & 3 & & & & 2.64518 & 2.49562 \\
\hline & & & & & 4 & & & & 2.66783 & 2.51842 \\
\hline & & & & & & 2 & & & 2.57982 & 2.42335 \\
\hline & & & & & & 3 & & & 2.54005 & 2.40060 \\
\hline & & & & & & & 2 & & 3.60562 & 3.24133 \\
\hline & & & & & & & 3 & & 4.77669 & 4.16816 \\
\hline & & & & & & & & 0.10 & 2.97829 & 2.66256 \\
\hline & & & & & & & & 0.15 & 3.37434 & 2.88136 \\
\hline
\end{tabular}


Table 3 Local Nusselt number $\left(\mathrm{Nu} / \mathrm{Re}_{\chi}\right)$

\begin{tabular}{|c|c|c|c|c|c|}
\hline$Q_{H}$ & $\gamma$ & $S$ & $\varphi$ & Cu-water & $\mathrm{TiO}_{2}$ - water \\
\hline 1 & 2 & 1 & 0.05 & 0.779717 & 0.735561 \\
\hline 2 & & & & 0.844371 & 0.810043 \\
\hline \multirow[t]{7}{*}{3} & & & & 0.890440 & 0.861594 \\
\hline & 3 & & & 0.927487 & 0.865671 \\
\hline & 4 & & & 1.024570 & 0.949662 \\
\hline & & 2 & & 0.983947 & 0.922745 \\
\hline & & 3 & & 1.117110 & 1.054870 \\
\hline & & & 0.10 & 0.844161 & 0.761746 \\
\hline & & & 0.15 & 0.900935 & 0.786225 \\
\hline
\end{tabular}

considerable for nanofluids. It is to be noted that the highest heat transfer rate is obtained for $\mathrm{Cu}$ due to high thermal conductivity compared to $\mathrm{TiO}_{2}$. These results are in good agreement with Hamad and Pop [31] (Table 4). Hence, simulation experiments have been carried out under physiological conditions, whose results can be directly compared with clinical data in the literature. System dynamics demonstrates itself to be a powerful and easy-to-use educational tool for biomedical engineering and sciences and is also able to explain the behavior of a physiological system.

\section{Conclusions}

Hall and ion slip effects on MHD free convective rotating flow of nanofluids in a porous medium past a moving vertical semi-infinite flat plate are investigated. The conclusions are made as the following. The resultant velocity decreases with an increase in Hartmann number, suction parameter, solid volume fraction of nanoparticles, and rotation parameter but an opposite effect is noticed for Hall and ion slip parameters, injection parameter, and permeability parameter. An increase in convection and solid volume fraction of nanoparticles led to increase the thermal boundary layer thickness but a reverse trend occurs for the heat source parameter. The skin friction coefficient increases with solid volume fraction of nanoparticles, the intensity of the magnetic field, suction parameter, and rotation parameter and reduces with Hall and ion slip parameters. Increasing values of convection parameter, heat source parameter, suction parameter, and solid volume fraction of nanoparticles are to increase the rate of heat transfer for both nanofluids $\mathrm{Cu}$ and $\mathrm{TiO}_{2}$. The conclusions unveil that the blow of thermal convection of nanoparticles has increased the temperature distribution, which helps in destroying the cancer cells during the drug delivery process. This model for studying

Table 4 Comparison of results for the local Nusselt number $\left(\mathrm{Nu} / \mathrm{Re}_{x}\right)$

\begin{tabular}{lllll}
\hline$Q_{H}$ & $S$ & $\varphi$ & $C u-$ water [31] & Cu-water, Present results $\gamma \rightarrow 0$ \\
\hline 1 & 1 & 0.05 & 0.688574 & 0.733261 \\
2 & & 0.744748 & 0.808269 \\
3 & & 0.856998 & 0.860109 \\
& 2 & 0.895578 & 0.919390 \\
& & 1.002578 & 1.051350 \\
& & 0.785549 & 0.787435 \\
& 0.10 & 0.884785 & 0.780187 \\
\hline
\end{tabular}


flow through porous media explains the development of bio-convection patterns generated by populations of gravitactic microorganisms in porous media.

\section{Nomenclature}

$u, v, w$ Velocity components along $x, y$ and $z$-axis respectively $(\mathrm{m} / \mathrm{s})$

$K_{n f}$ Thermal conductivity of nanofluid (W/m.K)

$U_{r}$ The uniform reference velocity $(\mathrm{m} / \mathrm{s})$

$g$ Acceleration due to gravity $\left(\mathrm{m} / \mathrm{s}^{2}\right)$,

$k$ Permeability of porous medium $\left(\mathrm{m}^{2}\right)$

$T$ Dimensional temperature (K)

$T_{w}$ Constant temperature (K)

$T_{\infty}$ Free stream temperature (K)

$Q$ Heat absorption coefficient $\left(\mathrm{W} / \mathrm{m}^{2} . \mathrm{K}\right)$

$w_{0}$ The normal velocity at the plate $(\mathrm{m} / \mathrm{s})$

$R$ Rotation parameter

$M$ Magnetic field parameter

Pr Prandtl number

$S$ Suction $(S>0)$ or injection $(S<0)$ parameter,

$K$ Permeability parameter

$Q_{H}$ Heat source parameter

$B_{0}$ Magnetic induction $(\mathrm{A} / \mathrm{m})$

$k$ Permeability of porous medium $\left(\mathrm{m}^{2}\right)$

$R e_{x}$ Local Reynolds number

$C_{p}$ Specific heat at constant pressure (J/Kg.K)

$\mathrm{Nu}$ Nusselt number

$n$ Frequency of oscillation $(\mathrm{Hz})$

$t$ Time (s)

$B$ Magnetic field vector $(\mathrm{A} / \mathrm{m})$

$E$ Electric field (c)

$V$ Velocity vector $(\mathrm{m} / \mathrm{s})$

$J$ Current density vector $\left(\mathrm{A} / \mathrm{m}^{2}\right)$

\section{Greek symbols}

$\beta_{n f}$ Coefficient of the thermal expansion of nanofluid $\left(\mathrm{K}^{-1}\right)$

$\Omega$ Angular velocity $(\mathrm{r} / \mathrm{s})$

$\theta$ Non-dimensional temperature

$\gamma$ Convective parameter

$\tau$ Skin friction

$\alpha_{n f}$ Thermal diffusivity of the nanofluid (WmK ${ }^{-1}$ )

$(\rho \beta)_{n f}$ The thermal expansion coefficient of the nanofluid

$\phi$ Solid volume fraction of the nanoparticles

$v_{f}$ Kinematic viscosity of nanofluid $\left(\mathrm{m}^{2} / \mathrm{s}\right)$

$\varepsilon$ Small constant quantity 
$\sigma$ Electrical conductivity of the fluid $(\mathrm{S} / \mathrm{m})$

$\rho_{n f}$ Density of the nanofluid $\left(\mathrm{Kg} / \mathrm{m}^{3}\right)$

$\mu_{n f}$ Viscosity of the nanofluid (Pa s)

$\left(\rho C_{p}\right)_{n f}$ Heat capacitance of the nanofluid $\left(\mathrm{J} \mathrm{K}^{-1}\right)$,

$\omega_{e}$ Cyclotron frequency $(\mathrm{e} / \mathrm{mB})$

$\tau_{e}$ Electron collision time (s)

$\beta_{i}$ ion slip parameter

$\beta_{e}$ Hall parameter

\section{Subscripts}

\section{$f$ Base fluid \\ $n f$ Nanofluid \\ $s$ Nanosolid particles}

\section{Acknowledgements}

The authors are grateful and express their sincere thanks to the editor, reviewers, and Team of Journal of Egyptian Mathematical Society for giving suggestions and the improvement of this paper.

\section{Authors' contributions}

MVK carried out the problem designing, performed the computational analysis, participated in the sequence alignment, and drafted the manuscript. AJC participated in its design and coordination and helped to draft the manuscript. The authors read and approved the final manuscript.

\section{Funding}

The authors declare that they have no funding.

\section{Availability of data and materials}

Data sharing is not applicable in this article as no datasets were generated or analyzed during the current study.

\section{Competing interests}

The authors declare that they have no competing interests.

\section{Author details}

'Department of Mathematics, Rayalaseema University, Kurnool, Andhra Pradesh 518007, India. ²Department of Mechanical Engineering, Prince Sultan Endowment for Energy and Environment, Prince Mohammad Bin Fahd University, Al-Khobar 31952, Kingdom of Saudi Arabia. ${ }^{3}$ RAK Research and Innovation Center, American University of Ras Al Khaimah, Ras Al Khaimah 10021, United Arab Emirates.

Received: 16 October 2019 Accepted: 19 December 2019

Published online: 06 January 2020

\section{References}

1. Choi, S.U.S.: Enhancing thermal conductivity of fluids with nanoparticles. Fluids Engg Div. 231, 99-105 (1995)

2. Vafai Kumbiz: Porous Media: Applications in biological systems and biotechnology. CRC Press. 1st Edition. 1-25 (2010), Taylor Francis Group, Boca Raton, Florida

3. Srinivasacharya, D., Shafeeurrahman, M.: Hall and ion slip effects on mixed convection flow of nanofluid between two concentric cylinders. J Asso Arab Uni Basic Appl Sci. 24(1), 223-231 (2017). https://doi.org/10.1016/j.jaubas.2017.03.002

4. Veera Krishna, M., Chamkha, A.J.: Hall and ion slip effects on MHD rotating boundary layer flow of nanofluid past an infinite vertical plate embedded in a porous medium. Results Phys. 15, 102652 (2019). https://doi.org/10.1016/j.rinp.2019. 102652

5. Veera Krishna, M., Chamkha, A.J.: Hall effects on MHD squeezing flow of a water based nano fluid between two parallel disks. J Por Med. 22(2), 209-223 (2019). https://doi.org/10.1615/JPorMedia.2018028721

6. Ram, P.C.: The effects of Hall and ion slip currents on free convective heat generating flow in a rotating fluid. Int. J. Energy Res. 19(5), 371-376 (1995). https://doi.org/10.1002/er.4440190502

7. Seddeek, M.A.: The effects of Hall and ion-slip currents on magneto-micropolar fluid and heat transfer over a nonisothermal stretching sheet with suction and blowing. Proc Royal Soc London A. 457, 3039-3050 (2001). https://doi.org/ 10.1098/rspa.2001.0847

8. Seddeek, M.A., Abdelmeguid, M.S.: Hall and ion-slip effects on magneto-micropolar fluid with combined forced and free convection in boundary layer flow over a horizontal plate. J Korea Soc Indu Appl Math. 8(2), 51-73 (2004)

9. Jha, B.K., Apere, C.A.: Combined effect of Hall and ion-slip currents on unsteady MHD couette flows in a rotating system. J Phys Soc Jpn. 79(10), 104401 (2010). https://doi.org/10.1143/JPSJ.79.104401

10. Uddin, Z., Kumar, M.: Hall and ion-slip effect on MHD boundary layer flow of a micro polar fluid past a wedge. Sci Iran B. 20(3), 467-476 (2013). https://doi.org/10.1016/j.scient.2013.02.013 
11. Ellahi, R., Bhatti, M.M., Pop, I.: Effects of Hall and ion slip on MHD peristaltic flow of Jeffrey fluid in a non-uniform rectangular duct. Int J Num Meth Heat Fluid Flow. 26(6), 1802-1820 (2016). https://doi.org/10.1108/HFF-02-2015-0045

12. Bhatti, M.M., Abbas, M.A., Rashidi, M.M.: Effect of Hall and ion slip on peristaltic blood flow of Eyring Powell fluid in a non-uniform porous channel. World J Model Simul. 12(4), 268-279 (2016)

13. Veera Krishna, M., Subba Reddy, G.: Unsteady MHD reactive flow of second grade fluid through porous medium in a rotating parallel plate channel. J. Anal. 27(1), 103-120 (2019). https://doi.org/10.1007/s41478-018-0108-3

14. Jitendra, K.S., Srinivasa, C.T.: Unsteady natural convection flow of a rotating fluid past an exponential accelerated vertical plate with Hall current, ion-slip and magnetic effect. Multidisci Model Mat Stru. 14(2), 216-235 (2018). https://doi.org/10. 1108/MMMS-06-2017-0045

15. Dillep Singh, C., Priyonk, R.: Hall current and heat transfer effects on MHD flow in a channel partially filled with a porous medium in a rotating system. Turk J Eng Env Sci. 33, 167-184 (2009). https://doi.org/10.3906/muh-0905-6

16. Veera Krishna, M., Subba Reddy, G., Chamkha, A.J.: Hall effects on unsteady MHD oscillatory free convective flow of second grade fluid through porous medium between two vertical plates. Phy Flu. 30, 023106 (2018). https://doi.org/10. $1063 / 1.5010863$

17. Veera Krishna, M., Chamkha, A.J.: Hall effects on unsteady MHD flow of second grade fluid through porous medium with ramped wall temperature and ramped surface concentration. Phy Flu. 30, 053101 (2018). https://doi.org/10.1063/1. 5025542

18. Veera Krishna, M., Swarnalathamma, B.V., Chamkha, A.J.: Heat and mass transfer on magnetohydrodynamic chemically reacting flow of micropolar fluid through a porous medium with Hall effects. Spe Top Rev Por Med An Inter J. 9(4), 347-364 (2018). https://doi.org/10.1615/SpecialTopicsRevPorousMedia.2018024579

19. Veera Krishna, M., Swarnalathamma, B.V., Chamkha, A.J.: Investigations of Soret, Joule and Hall effects on MHD rotating mixed convective flow past an infinite vertical porous plate. J Ocean Engg Sci. 4(3), 263-275 (2019). https://doi.org/10. 1016/j.joes.2019.05.002

20. Veera Krishna, M., Bharathi, K., Chamkha, A.J.: Hall effects on MHD peristaltic flow of Jeffrey fluid through porous medium in a vertical stratum. Interfacial Phen Heat Transf. 6(3), 253-268 (2019). https://doi.org/10.1615/ InterfacPhenomHeatTransfer.2019030215

21. Sara, I.A., Bhatti, M.M.: The study of non-Newtonian nanofluid with Hall and ion slip effects on peristaltically induced motion in a non-uniform channel. RSC Adv. 8, 7904-7915 (2018). https://doi.org/10.1039/c7ra13188g

22. Bhatti, M.M., Ellahi, R., Zeeshan, A., Marin, M., Ijaz, N.: Numerical study of heat transfer and Hall current impact on peristaltic propulsion of particle-fluid suspension with compliant wall properties. Mod Phy Let B. 1950439 (2019). https:// doi.org/10.1142/s0217984919504396

23. Waqas, H., UllahKhan, S., Hassan, M., Bhatti, M.M., Imran, M.: Analysis on the bioconvection flow of modified second grade nanofluid containing gyrotactic microorganisms and nanoparticles. J Mol Liq. 291, 111231 (2019). https://doi.org/ 10.1016/j.molliq.2019.111231

24. Sheikholeslami, M., Bhatti, M.M.: Influence of external magnetic source on nanofluid treatment in a porous cavity. J Por Med. 22(12), 1475-1491 (2019). https://doi.org/10.1615/JPorMedia.2019024518

25. Veera Krishna, M., Anand, P.V.S., Chamkha, A.J.: Heat and mass transfer on free convective flow of a micro-polar fluid through a porous surface with inclined magnetic field and Hall effects. Spe Top Rev Por Med An Inter J. 10(3), 203-223 (2019). https://doi.org/10.1615/SpecialTopicsRevPorousMedia.2018026943

26. Veera Krishna, M., Chamkha, A.J.: MHD peristaltic rotating flow of a couple stress fluid through a porous medium with wall and slip effects. Spe Top Rev Por Med An Inter J. 10(3), 245-258 (2019). https://doi.org/10.1615/ SpecialTopicsRevPorousMedia.2019028609

27. Oztop, H.F., Abu-Nada, E.: Numerical study of natural convection in partially heated rectangular enclosers with nanofluids. Int J Heat Fluid Flow. 29, 1326-1336 (2008). https://doi.org/10.1016/j.jigheatfluidflow.2008.04.009

28. Sutton, G., Sherman, A.: Engineering magnetohydrodynamics. Mc Graw Hill, New York (1965)

29. Maxwell, J.: A treatise on electricity and magnetism, 2nd edn. Oxford University Press, Cambridge (1904)

30. Brinkman, H.C.: Viscosity of concentrated suspensions and solution. J Chem Phys. 20, $571-581$ (1952)

31. Hamad, M.A.A., Pop, I.: Unsteady MHD free convection flow past a vertical permeable flat plate in a rotating frame of reference with constant heat source in a nanofluid. Heat Mass Transf. 7, 1517-1524 (2011). https://doi.org/10.1007/ s00231-011-0816-6

32. Ganapathy, R.: A note on oscillatory Couette flow in a rotating system. ASME J Appl Mech. 61, 208-209 (1994). https:/ doi.org/10.1115/1.2901403

\section{Publisher's Note}

Springer Nature remains neutral with regard to jurisdictional claims in published maps and institutional affiliations. 